\title{
Rule-based system to assist a powered wheelchair driver
}

\author{
David A Sanders \\ Engineering (School of) \\ Portsmouth University \\ United Kingdom \\ david.sanders@port.ac.uk \\ Alexander Gegov \\ Computing (School of) \\ Portsmouth University \\ United Kingdom \\ alexander.gegov@port.ac.uk
}

\author{
Giles E Tewkesbury \\ Engineering (School of) \\ Portsmouth University \\ United Kingdom \\ giles.tewkesbury@port.ac.uk
}

\author{
Rinat Khusainov \\ Engineering (School of) \\ Portsmouth University \\ United Kingdom \\ rinat.khusainov@port.ac.uk
}

\begin{abstract}
Some rule based techniques are presented to assist powered wheelchair drivers. A driver is included as much as possible while a sensor system assists by avoiding obstacles and tending to turn to point towards a specified destination. A generated angle to turn towards the destination of the wheelchair is added as an extra input. Other inputs are from a joystick and from sensors. Recommended directions are suggested and they are mixed with speed and direction inputs from the joystick. An angle to turn the powered wheelchair is suggested by the rulebased system. The suggested angle is then mixed with the other inputs from the sensors and the joystick. A revised direction for the wheelchair is generated and that helps a wheelchair user to drive their powered wheelchair.
\end{abstract}

Keywords-Wheelchair; Powered; Assist; Rule-based, Driving; Collision Avoidance.

\section{INTRODUCTION}

This paper proposes a rule based system to assist with the control of a powered wheelchair. Powered wheelchairs can be useful for disabled people who cannot turn the wheels of a manual wheelchair. A powered wheelchair can bring freedom and independence to people who are relying on others [1]. The methods described in this paper will help more disabled people to successfully drive a powered wheelchair. Ultrasonic sensors provide knowledge about the local environment around a powered wheelchair while being driven towards a destination. The system can then assist the disabled driver with avoiding obstacles and obstructions in their path and with successfully moving towards their destination.

About 7 million Americans use assistive mobility devices. There are about 2 million scooter or wheelchair drivers and about 5 million people use other devices, for example crutches, walkers and canes [2]. Nearly 1/3 of the people using mobility devices also require the assistance of other people [1].
Osteoarthritis and stroke and the predominant primary conditions for scooter and wheelchair drivers. Osteoarthritis is the dominant condition associated with the use of mobility devices[1-2].

A powered wheelchair is usually used by people lacking dexterity or mobility because of hand, arm, shoulder or more universal disabled condition, and who don't have the strength in their legs to use their feet to push a manual wheelchair. Powered wheelchairs can also provide elevation, recline, tilt, and other bespoke functions required or useful to functioning and health.

There are four typical powered wheelchairs: those driven by the rear, center or front wheels and those driven by all four wheels. Powered wheelchairs can also be divided by their type of seat: (a) similar to manual wheelchairs, with a sling-style frame and seat, or (b) with a seat similar to a car.

A wheelchair driver typically controls their direction and speed using a joystick. If a user does not have the required coordination to successfully use a joystick or if they cannot use their fingers and / or hands then there are other input devices that might be used (puff / sip tubes, switches, chin or head controllers, or foot control etc).

Until recently, powered wheelchairs were mainly for use indoors, but this is changing as technology has changed. Some powered wheelchairs are now equally suited to outdoor or indoor use.

Powered wheelchairs move at speeds of up to 6 m.p.h and some come with outdoor tires and wheels. They can also have extra supplementary wheels to make the wheelchair more stable, for example when they are being used outside and away from roads or pavements. Mid or rear wheel drive powered chairs are popular for use both outdoors and indoors.

Powered wheelchair users can spend a lot of time in their chairs, so the chair needs to suit the environment where it is 
used. Each driver is unique. Diverse users need diverse seats, leg rests or arm rests to provide them with stability and comfort. Powered seats, tilting and reclining backs, or electric leg rests are optional accessories.

If a user has physiological or neurological problems, head injuries or lacks special awareness then a potential powered wheelchair user might be unable to safely steer a wheelchair. A potential driver might be blind, lack special awareness or be unable to avoid collisions. The systems described in this paper help disabled wheelchair users to drive more safely in those circumstances.

Wheelchair controllers are usually open-loop and drivers indicate their desired direction and speed by repositioning an iput device such as a joystick so that the wheelchair tends to move along the indicated route at the desired speed. Drivers correct their route to avoid obstacles. In the work described here, information from the input device is processed and mixed with the inputs from the sensors and a destination in an effort to assist a driver in guiding their wheelchair. Local and global planning are blended within the rule-based system. The output assists drivers with their task. Local input from sensors is mixed with a global path [3].

The navigation of powered wheelchairs has been investigated [4-5]. Algorithms have typically been local and no attempt was made to improve the systems in any sort of global way. Obstacle avoidance has been considered as an optional add-on for them [6] together with the use of local inputs from sensors [7].

Some research has planned preliminary paths for wheelchairs and then modified them locally when obstructions were perceived [3] but those systems have hardly ever been used effectively to assist wheelchair drivers. In the work described in this paper, a local planner drives the motors attached to the wheels subject to three inputs. They are from on-board sensors mounted on the wheelchair, from the wheelchair joystick, and from a more global destination. The wheelchair reacts quickly to movements of the joystick but also reacts to obstacles detected ahead of the wheelchair. The wheelchair avoids obstacles but always tends to turn in the direction of the global destination.

Huq et al. eliminated some limitations experienced with previous methods[8] by using a fuzzy context-dependent blending of schemas using goal oriented navigation while also avoiding objects in the wheelchair path. Fuzzy logic was mixed with genetic algorithms to overcome mapping problems and to determine local position [9]. Bennewitz and Burgard described random planning techniques that were able to produce real time paths in unfamiliar surroundings [3], [10], that could precisely follow a trajectory [11]. Hwang and Chang produced an obstacle avoidance method using fuzzy decentralized slidingmode control [12]. Song and Chen solved some local minima problems and enhanced the potential-field-method [7] and Nguyen et al created some Bayesian Neural Networks that could be used to avoid obstacles [13].

Research described in this paper presents techniques to partly optimise minimum-cost routes towards a destination. A joystick mostly regulates speed and direction, but a simple AI system also provides and input [14-17]. Perception based rules

Identify applicable sponsor/s here. If no sponsors, delete this text box (sponsors). are used that are similar to those used by Sanders in [35] and Parhi \& Singh in [3].

Algorithms trade path length against distance to objects. Rules are used to determine a suggested steering angle. That steering angle is merged with an input from a joystick and the new steering angle is used to produce motor drive signals. The techniques desribed in the paper were tested using a simulation and using a Bobcat II wheelchair (Fig. 1) with sensors mounted onto the chair.

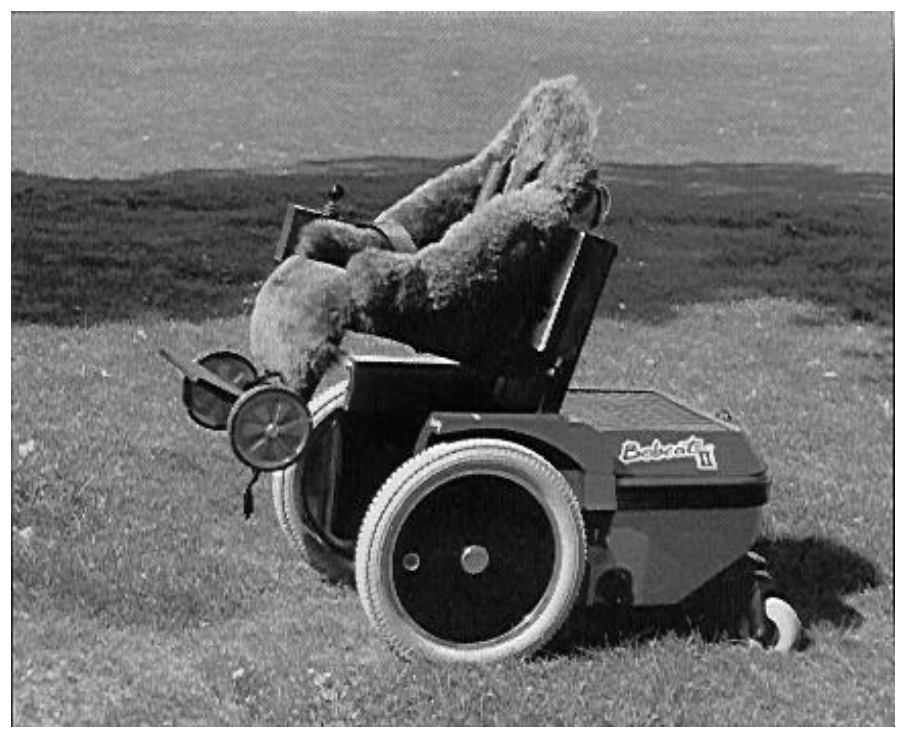

Fig. 1 Bobcat II wheelchair

A wheelchair should safely avoid objects in the path of the chair [18]. Many sensors can be used to assist a wheelchair driver in avoiding objects: structured light or laser [19]; ultrasonics [20]; or infrared [21]. It can be a problem to use global systems inside a building [22] but simpler local sensor based systems have been used: tilt sensors, odometers, gyroscopes, and ultrasonic sensors [23, 24]. Cameras are getting cheaper and have been used but their processing systems tend to be more complicated and use more processing power [25]. Computers are becoming more and more powerful as well as getting cheaper [26] so that cameras may be used more on wheelchairs in the future. The disabled human being driving the wheelchair is still usually the best source of knowledge about what they want to do, the wheelchair environment and the situation but their disability and reduced visibility can diminish their ability [27].

Ultrasonic sensors were selected in this work because they were simple, cheap and robust [28]. The input from the sensors and from the joystick are described in Part II and Part III presents the wheelchair kinematics and Part IV presents rues and wheelchair control. Part V describes some of the tests and presents results and Part VI completes the paper with brief conclusions.

\section{SENSORS AND JOYSTICK INPUT}

\section{A. Ultrasonic sensors}

Ultrasonic sensors and systems were similar to those in [29$31,35]$. A sensor was mounted above each of the driving wheels 
at the front of the wheelchair. A measure of distance to objects was then provided by measuring the time taken for a pulse to reflect back and reach the receiver on the powered wheelchair.

The BobCat II wheelchair had a substantial steel framework for stability and strength that was covered with a fibreglass shell. The large driving wheels are at the front of the wheelchair and there were trailing casters at the rear. An ultrasonic sensor was secured above each driving wheel.

The powered wheelchair was steered by a joystick that was normally connected directly to the wheelchair controller. In this work, the direct connection between the joystick and the wheelchair controller was separated and a computer was inserted between them. The computer managed the joystick input. The whole system was able to function in two ways:

1. The input signal from the joystick was sent straight to the wheelchair controller.

2. The computer could modify the speed and direction of the powered wheelchair.

The modification of the speed and direction adhered to the following three basic rules:

1. The disabled human driver remained in overall control.

2. The sensors only changed the speed and direction when they needed to (for example if an obstacle was detected ahead of the wheelchair).

\section{Changes of direction and speed were realisable.}

If an object was within range of the sensors then an iimaginary potential field was effectively placed around it $[7,23$, 35]. If obstacles were not being sensed by the system then the range-finder progressively increase range ( by lengthening pulses) until a potential obstacle was detected and that gave earlier warnings of likely difficulties.

\section{B. The mapping of the environment to the wheelchair front}

Ultrasonics can give false readings and be noisy. Histogramic In-Motion Mapping filtered out false readings [35]. The volume to the front of the powered wheelchair was split into right hand and left hand matrices, with NEARBY, MIDWAY and DISTANT, elements within them. When an object was perceived forward of the wheelchair then it was classified as NEARBY, MIDWAY or DISTANT. The beams from the sensors over-lapped and enclosed the volume to the front of the wheelchair. So, in addition to the right hand and left hand matrices, a central matrix represented situations when both right hand and left hand sensors sensed an obstacle.

The volume of space to the front of the wheelchair was represented by nine elements in a 3 x 3 two dimensional grid:

\section{LEFT HAND, CENTRE, RIGHT HAND $x$}

\section{NEARBY, MIDWAY, DISTANT}

That was a grid of nine cells. When an object was detected then the element(s) within the grid that were associated with detection were stepped up with a comparatively large value, for example 6, up to a maximum value of 16 . Other cells reduced by lesser quantities (for example 2) to zero. That created a simple histogrammic representation of the volume in front of the wheelchair. If an object was detected in a cell then that cell rapidly stepped up in value. Any random misreads in any of the other cells would briefly increase for the solitary misread and then would quickly reduce. If an obstacle was detected in one cell but then moved into another cell, then the new cell rapidly stepped up in value. When the obstacle vanished from the first cell then it's value stepped down to zero. Reliable ranges were arrive at in $<0.5$ seconds.

\section{Joystick interpretation}

The powered wheelchair was fitted with a Penny \& Giles joystick. It contained two potentiometers and the position of the joystick was determined using a pair of $\mathrm{A} / \mathrm{D}$ converters connected to the potentiometers. Data from the joystick was in Cartesian coordinates. These were converted into polar coordinates: $|\mathrm{J}| \angle \theta$. Desired speed was characterised by the amount that the joystick was moved away from the central rest position. That amount was represented by $|\mathrm{J}|$. The desired direction was represented by the angle $\angle \theta$.

The amount of time in seconds that the joystick remained in the same position implied a level of confidence that the operator had in their judgement.

$$
|\mathrm{J}|=\sqrt{ }((\mathrm{JA} * \mathrm{JA})+(J \mathrm{~B} * \mathrm{~J} B))
$$

Where JA and JB are Cartesian co-ordinates.

The histogrammic depiction worked as a pseudo-integrator. If the joystick was held in the same position, then the cell that was associated with that position stepped up in value over time and other cells stepped down in value. The cell with the largest value represented the position of the joystick.

$|\mathrm{J}|$ and $\theta$ established joystick position and therefor desired speed and direction. Position and confidence in that position were both recorded. Each cell in the array had two values:

- "AngularConfidence" showed a steady joystick position.

- "Magnitude" showing the desired wheelchair speed.

JoystickIn was an input to the rule based system. JoystickIn provided a confidence-level of the intention of the disabled driver. JoystickArray was a computer procedure that calculated which cell represented the joystick and AngularConfidence stepped up. Other empty cells stepped down. Histogram elements rapidly reduced in value but increased in value more slowly. JoystickArray cells stepped up to a maximum value in $<0.5 \mathrm{~s}$ and stepped down to zero in $<140 \mathrm{~ms}$.

The rates to step up or step down values were established experimentally. Different weights could be set for different disabled users or for specific tasks.

\section{BOBCAT II WHEELCHAIR KINEMATICS}

The kinematics of the powered wheelchair (Fig. 1) are explained. The Bobcat II wheelchair has a pair of larger independent driving wheels positioned at the front of the wheelchair.

Turning the driving wheels at different independent speeds allowed a disabled driver to control the speed and direction of 
the powered wheelchair. If the radius of the driving wheels is $r$ then diameter is 2r. Employing symbolisation and notation from [3] and [35], driving wheels were distance $\mathrm{W}$ apart. $\mathrm{C}$ is centre of gravity for the wheelchair. $\mathrm{P}$ is at the meeting point of a line through the wheelchair centre and a line through the wheel axis. The distance between $\mathrm{C}$ and $\mathrm{P}$ is $\mathrm{d}$.

Kinematics for a Bobcat II powered wheelchair are shown in Fig. 2.

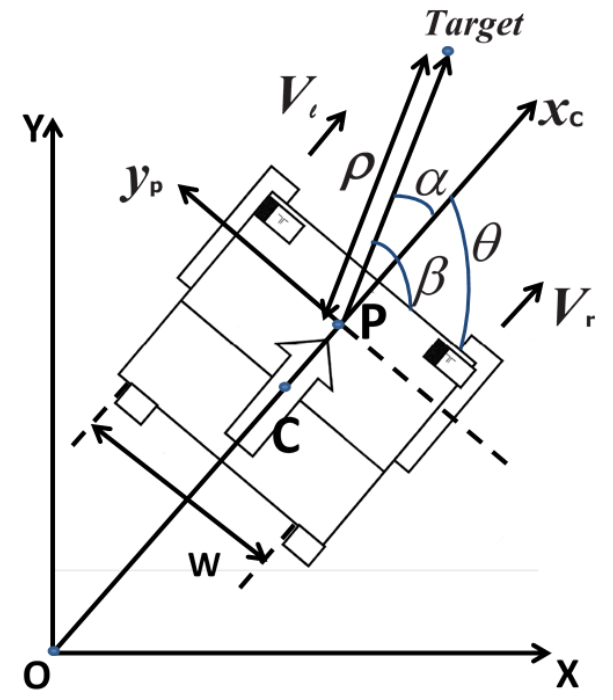

Fig 2 The kinematics of the powered wheelchair

It was presumed that there was not any slip between the floor and the wheels.

$$
\begin{aligned}
& v_{\text {tang }}=1 / 2\left(v_{\text {right }}+v_{\text {left }}\right) \\
& \omega_{\text {tang }}=1 / \mathrm{W}\left(v_{\text {right }}-v_{\text {left }}\right) \\
& v_{\text {right }}=r \omega_{\text {right }} \text { and } v_{\text {left }}=r \omega \text { left }
\end{aligned}
$$

where $v$ is linear velocity and $\omega$ is the angular velocity of the powered wheelchair.

Using vector notation, $[\mathrm{O} \mathrm{X} \mathrm{Y}]$ is the global position of the powered wheelchair in global coordinates. That is:

$$
\boldsymbol{q}=\left[\mathrm{xc} \mathrm{y}_{\mathrm{P}} \theta\right]^{\top}
$$

where $\mathrm{y}_{\mathrm{P}}$ and $\mathrm{X}_{\mathrm{C}}$ are the global coordinates of the meeting point $P$. $\theta$ is the orientation of the local coordinate frame of the wheelchair, that is $\left[P X_{C} Y_{P}\right]$ in Fig. 2. established from the horizontal axis. The configuration of the wheelchair is defined by the coordinates (5). The driving wheels on the powered wheelchair are assumed not to slip and the body is assumed to be rigid. That means that the powered wheelchair can only move in a direction that is normal to the axis of the wheels. And, the velocity at the point of contact between the ground and the wheel (orthogonal to the plane of the wheel) is zero.

$$
\left(d y_{p} / d t\right) \cos \theta-(d x c / d t) \sin \theta-d \theta / d t=0
$$

Kinematics restrictions are not time dependent and are

$\mathrm{A}^{\top}(\boldsymbol{q}) \mathrm{d} \boldsymbol{q} / \mathrm{dt}=0$

where $C^{\top} A(\boldsymbol{q})=0$

and $A(\boldsymbol{q})$ is an input matrix associated with the constraints.

where $C(\boldsymbol{q})$ is a full-rank matrix formed by a set of linearly independent vector fields covering the null space of $A^{\top}(\boldsymbol{q})$. $V$ tang is a vector time function that can be found from equations (7) and (8) for times t.

$\mathrm{d} \boldsymbol{q} / \mathrm{dt}=\mathrm{C}(\boldsymbol{q}) \boldsymbol{v}$ tang

The constraint matrix in (6) for the powered wheelchair is

$\mathbf{A}^{\top}(\boldsymbol{q})=[-\sin \theta \cos \theta-\mathrm{d}]$

and

$\boldsymbol{V}_{\text {tang }}=\left[\begin{array}{ll}v & \omega\end{array}\right]^{\top}$

Where $\mathrm{v}$ is linear velocity and $\omega$ is angular velocity of the meeting point $\mathrm{P}$ (taken along the powered wheelchair axis).

So, the kinematics described by (9) can be portrayed in a $\mathrm{d} q / \mathrm{dt}$ matrix.

If the powered wheelchair is considered to only move forward and not to reverse, then $v=-v$, ang and a new simplified matrix can represent the wheelchair. determined

A controller generated the steering angle and wheel velocities where Angle $=\left(v_{\text {left }}-v_{\text {right }}\right) / \mathrm{W}$, to steer the powered wheelchair along the desired path.

\section{THE RULES AND CONTROL}

The required linear and angular velocities ( $v$ and $\omega)$ to move the powered wheelchair were determined such that they that would take the powered wheelchair from a current position $\left(\rho_{0} \alpha_{0} \beta_{0}\right)$ to a target position. Considering the use of linear control [34], then

$v=K_{\rho} \rho$

$\omega=K_{\alpha} \alpha+K_{\beta} \beta$

A matrix could depict the closed-loop system to drive the wheelchair to the destination $(\rho, \alpha, \beta)=(0,0,0)$.

The controller was coded with an overdamped system response and successfully simulated before being placed on the wheelchair. Input from the ultrasonic sensors was combined with the joystick input using rules intended to avoid obstacles. The rules were improved later by including the more global destination (the final destination or a via point along the way) so that the disabled drivers could easily follow the efficient global 
path. Five inputs were combined within the rules so that obstacles were avoided (fig 4). They were:

- $\quad$ Desired steering angle (obtained from the joystick);

- Distance to obstacles detected by both sensors;

- Distance to obstacles on the right of the wheelchair;

- Distance to objects on the left of the wheelchair.

- Target.

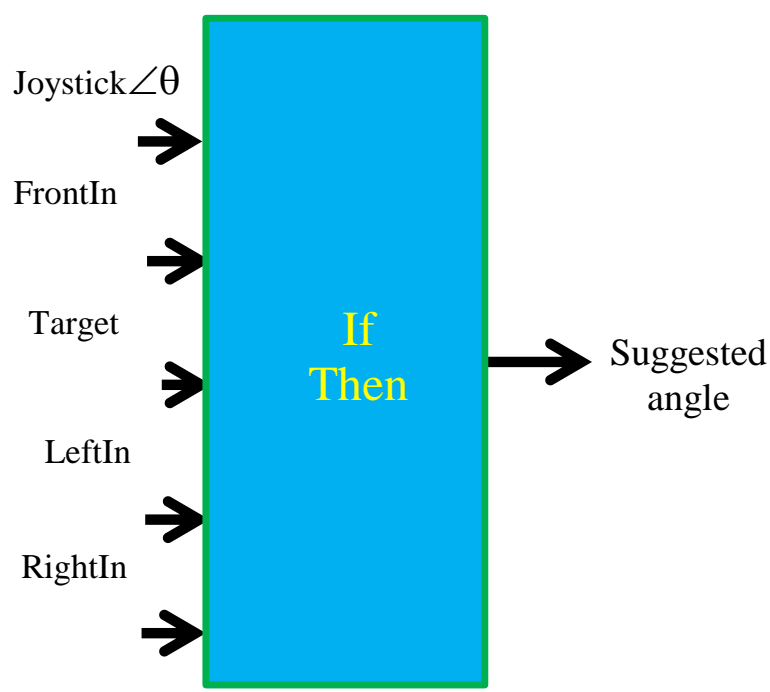

Fig. 3. Rule-based system.

The inputs form the ultrasonic sensors described the volume ahead of the powered wheelchair. Those inputs were used to adjust the steering angle fed to the controller. The result was a suggested trajectory that was efficient and safe for powered wheelchair movement. If $\angle \theta$ was left of the powered wheelchair then it tended to turn anticlockwise but if $\angle \theta$ was right of the powered wheelchair then it turned clockwise. The control systems worked well to improve function if human senses were impaired.

In addition to knowledge of the joystick steering angle and some knowledge about the volume ahead of the wheelchair, the rule based system also had a target destination. Including a target destination considerably increased the number of rules. As examples, some of the rules are described:

EXAMPLE ONE - the obstacle and destination are on left of the powered wheelchair:

Rule One: If Joystick $=0^{\circ}$ and LeftIn=MIDWAY and RightIn $\leq$ DISTANT and FrontIn $\leq$ DISTANT and AngleToTarget $=75^{\circ}$, then adjust steering angle by $0^{\circ}$

Rule Two: If Joystick $=0^{\circ}$ and LeftIn=MIDWAY and RightIn $\leq$ DISTANT and FrontIn $\leq$ DISTANT and AngleToTarget $=60^{\circ}$, then adjust steering angle by-10

Rule Threee: If Joystick $=0^{\circ}$ and LeftIn=MIDWAY and RightIn $\leq$ DISTANT and FrontIn $\leq$ DISTANT and AngleToTarget $=50^{\circ}$, then adjust steering angle by $-25^{\circ}$

EXAMPLE TWO - the obstacle and destination are on the right of the powered wheelchair:
Rule Four: If Joystick $=0^{\circ}$ and LeftIn $\leq$ DISTANT and RightIn = MIDWAY and FrontIn $\leq$ DISTANT and AngleToTarget $=75^{\circ}$, then adjust steering angle by $15^{\circ}$

Rule Five: If Joystick $=0^{\circ}$ and LeftIn $=\leq$ DISTANT and RightIn $=$ MIDWAY and FrontIn $\leq$ DISTANT and AngleToTarget $=60^{\circ}$, then adjust steering angle by $30^{\circ}$

Rule Six: If Joystick $=0^{\circ}$ and LeftIn $=\leq$ DISTANT and RightIn $=$ MIDWAY and FrontIn $\leq$ DISTANT and AngleToTarget $=30^{\circ}$, then adjust steering angle by $25^{\circ}$

EXAMPLE THREE - an obstacle is in front and the destination is on the right:

Rule Seven: If Joystick $=0^{\circ}$ and LeftIn $=$ NEARBY and RightIn = NEARBY and FrontIn $\leq$ DISTANT and AngleToTarget $=20^{\circ}$, then adjust steering angle by $15^{\circ}$

Rule Eight: If Joystick $=0^{\circ}$ and LeftIn $=$ NEARBY and RightIn $=$ NEARBY and FrontIn $\leq$ DISTANT and AngleToTarget $=25^{\circ}$, then adjust steering angle by20 0

Rule Nine: If Joystick $=0^{\circ}$ and LeftIn $=$ NEARBY and RightIn $=$ NEARBY and FrontIn $\leq$ DISTANT and AngleToTarget $=30^{\circ}$, then adjust steering angle by $25^{\circ}$

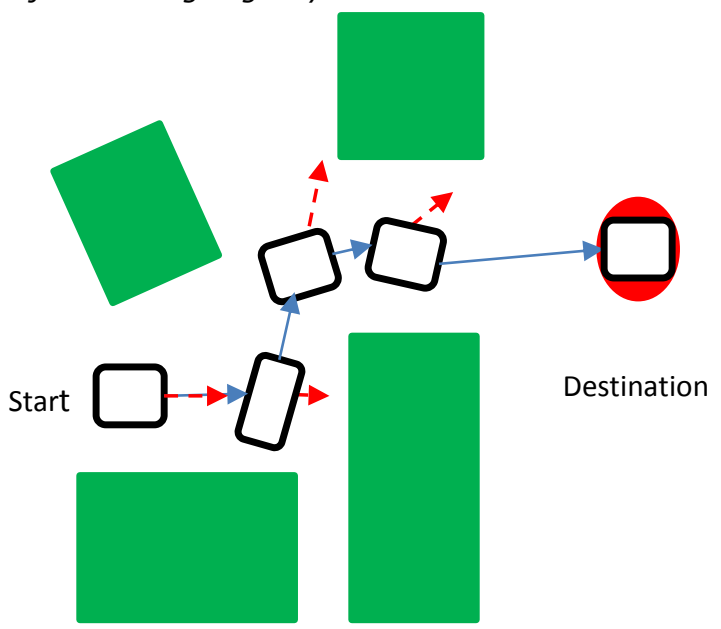

Fig. 4 Wheelchair using the rule set to move through a set of obstacles showing calculated directions (red dashed line) and approach directions (blue solid line).

The wheelchair system worked well using the rule set and especially assisted drivers when the human operator was impaired in some way. The path of the wheelchair is shown again in Fig. 4. The additional green solid arrow is pointing at the target destination. 


\section{TESTS AND THE RESUlTS}

An example of a simulation is displayed in Fig. 5.

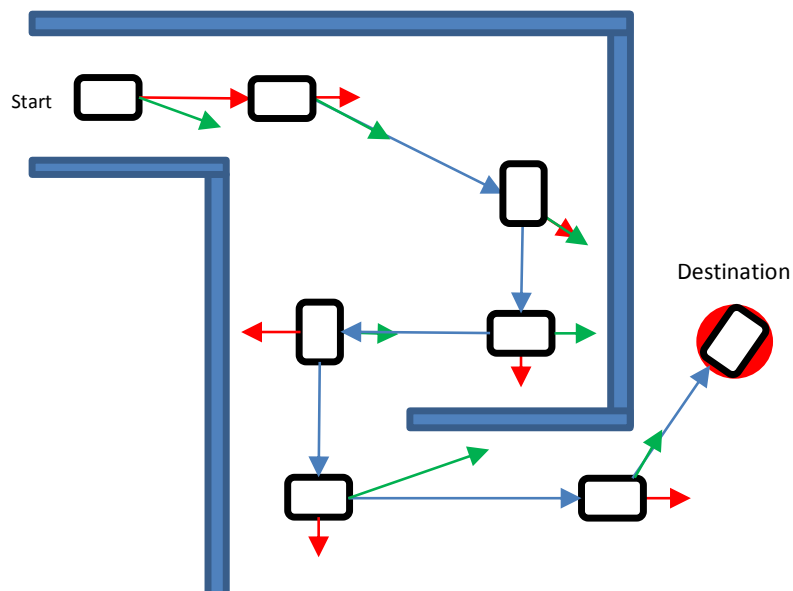

Fig. 5 An example of a simulation showing the wheelchair avoiding local minima (inner wall corners).

After the algorithms had been successfully tested in simulation, the hardware and software were mounted onto the powered wheelchair. A standard course at Portsmouth University was used for each test.

When the ultrasonic sensors detected an obstacle relatively close to the wheelchair, then the wheelchair turned away to avoid collision. That avoidance manoeuvre could be overruled by the wheelchair user sig their joystick if the user needed the wheelchair to move closer to the object. For example to move close to a wall to turn on a light switch.

If sensors detected an obstacle that was DISTANT or closer then avoidance was activated. If the ultrasonic sensors detected an object in front of the wheelchair while it was moving towards the target destination, then the powered wheelchair tended to turn and drive alongside the obstacle. When noting was in the way and the joystick was held in a forward position, then the

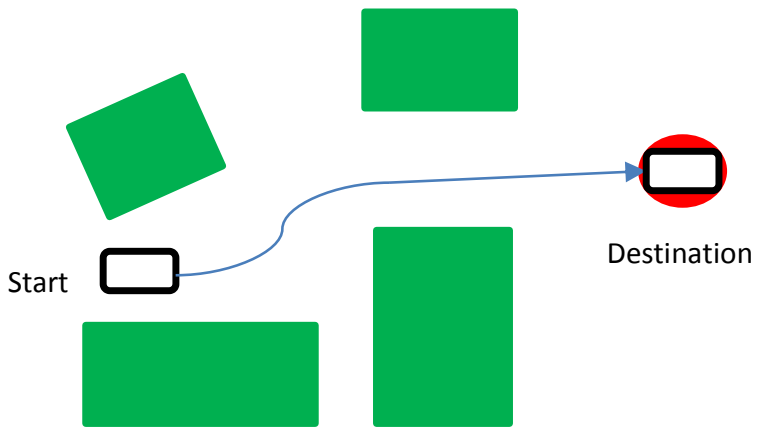

Fig. 6 Results from a real time experiment with the same rules applied.

powered wheelchair tended to head towards the target destination. That tended to decrease the time to reach the target destination when disability made steering difficult or if vision was impaired. The system turned the wheelchair so that the steering bearing changed and the wheelchair drove toward the destination.
Results from the simulation and from a real-time test with the powered wheelchair are in Fig. 6 and Fig 8.

Results from tests were compared with results described in [3]. The rule-based system described in this paper tended to perform faster. Figure 7 shows a comparison of time taken by the wheelchair systems as the powered wheelchair was driven through a set of standard test environments at Portsmouth University.

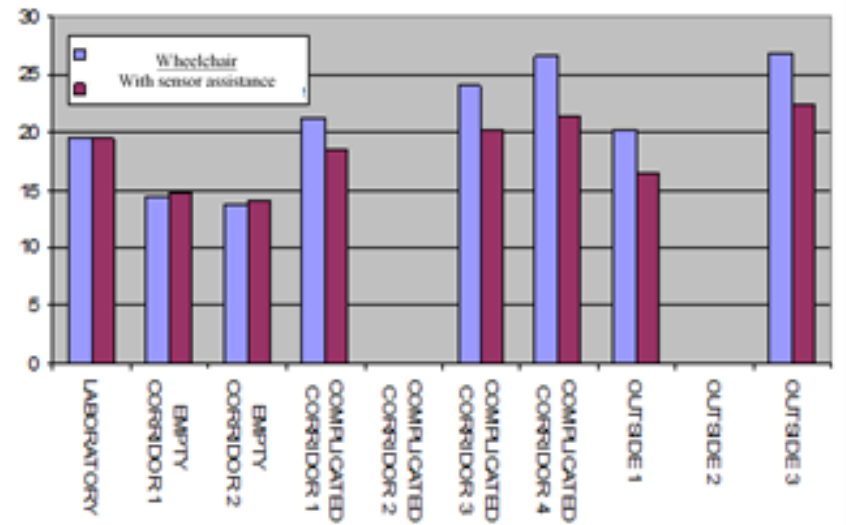

Fig. 7 Average time taken to complete set courses. Right hand (darker) bars show time taken with sensors used to assist a wheelchair driver and left hand (lighter) bars show time taken without any sensors being used to assist.

In most cases the average time taken to safely drive a route was less for the new wheelchair systems described here. Figure 7 contains two anomalies. The powered wheelchairs moved more quickly through the empty corridors without any sensors to assist the driver. As the routes became more complicated, then the sensor systems helped the wheelchair drivers.

Adding the target destination as an extra input to te rule based system did make driving less efficient in the easier parts of a route. In those cases, if the driver could see and understand what was happening then they did not need sensors to help them. For example, routes are shown in Fig. 8 and Fig. 9.

In Fig. 8, the wheelchair tended to be pulled towards the destination by the application of the rules.

The powered wheelchairs were able to reach destinations efficiently and if a user was capable of steering a wheelchair then they could overcome any application of the rules that might make a route less efficient.

Methods and techniques described in this paper produced quicker responses in most of the cases and reduced computation time needed when evaluated against other approaches. The rulebased system performed safely and effectively. 


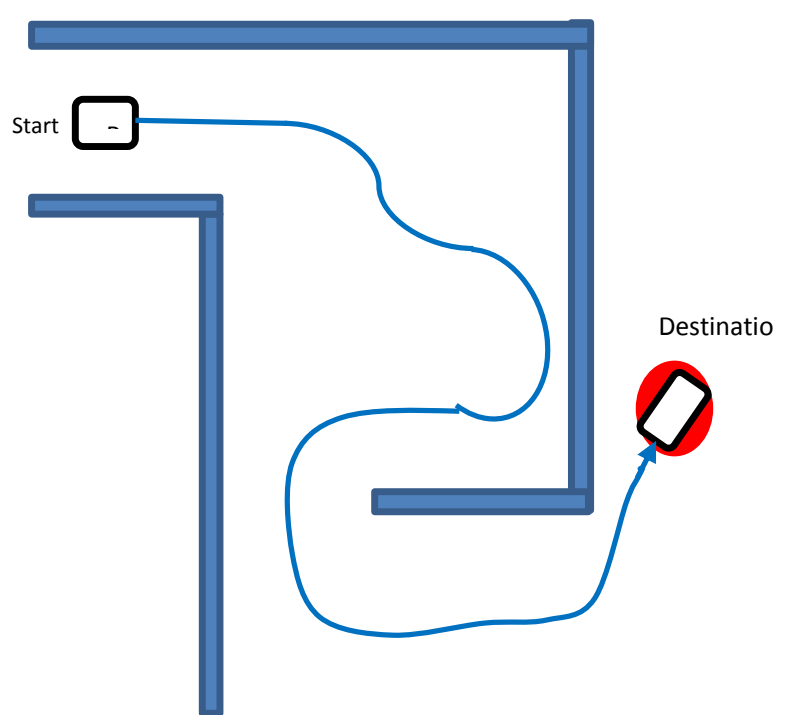

Fig 8. The path of a wheelchair using the rules when the driver is not able to drive to the destination without assistance.

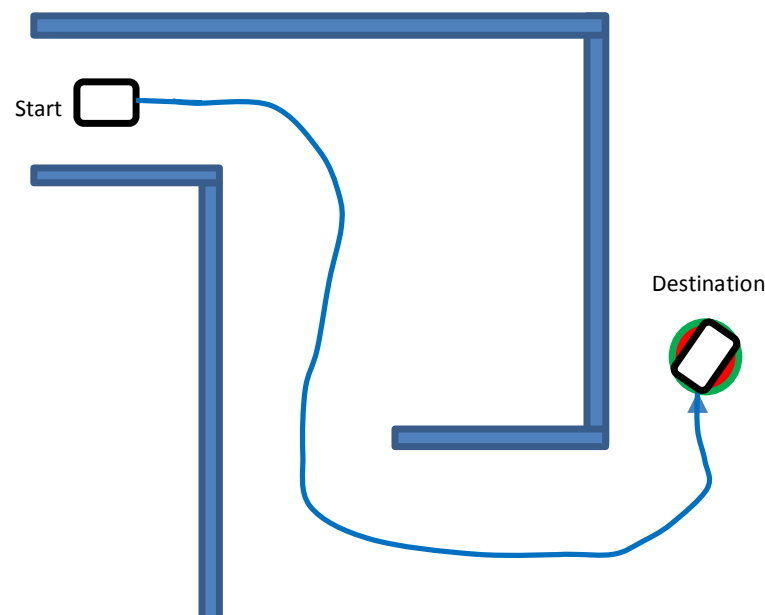

Fig 9. The path of a wheelchair when the driver is able to drive themselves to the destination.

The powered wheelchair needed to avoid moving and stationary objects (for example other humans standing or walking near to the powered wheelchair).

When sensors detected an obstacle near to the powered wheelchair, then the wheelchair turned away to avoid collision.

Avoiding collisions is a relatively high priority for powered wheelchairs. Collision avoidance tended to override the systems but if the joystick stayed still (roughly) in the same place then that input integrated over time so that the wishes if the disabled driver eventually overrides all other behaviours.

Collision avoidance was activated when the input from the sensor systems increased beyond a threshold limit set within the array cells.

When an obstacle was detected in front of the powered wheelchair while it is moving towards the destination then the wheelchair tended to follow the wall; the wheelchair rotates to move parallel to an obstacle edge or wall.
When nothing was being detected then the wheelchair tended to drive in a direction that was between the angle requested by the joystick and the angle to the target destination.

Results from the various tests were evaluated against other recent systems and these rule-based systems performed satisfactorily.

\section{Discussion AND CONCLUSION}

Rule-based systems described inn this paper proved to be sufficiently robust and safe. They are efficient and simple in assisting a wheelchair driver in steering a powered wheelchair.

The rule based techniques were successfully used to assist wheelchair drivers. The wheelchair system quickly detected objects and steered the wheelchair around them so that wheelchair users were assisted in completing driving tasks.

Laboratory and field testing was compared with simulations and rules were validated.

The systems described here were favourably compared with recent published systems and that also validated the methods and systems.

A limitation is that rules are hard-coded and cannot learn. That may become a limitation in the future and current work is investigating the use of ANNs for that learning. Current work is also investigating the mixing of new AI tools [35-46] so that specific tools can be used to best effect.

\section{REFERENCES}

[1] Electric Wheelchairs: Types \& Reviews of Powerchairs, Disabled World http://www.disabled-world.com/assistivedevices/mobility/wheelchairs

[2] Mobility Device Statistics - United States - The University of California - Disability Statistics Center - (2013-04-22). http://www.disabledworld.com/disability/statistics/mobility-stats.php

[3] D.R. Parhi, and M.K. Singh "'Rule-based hybrid neural network for navigation of a wheelchair," Proc. IMechE Part B: J. Engineering Manufacture, Vol. 224, 2009, pp 11103-1117.

[4] A.V. Nguyen, L.B. Nguyen, S. Su, and H.T. Nguyen "Shared Control Strategies for Human - Machine Interface in an Intelligent Wheelchair," 35th Annual Int Conf of IEEE-Engineering-in-Medicine-and-BiologySociety (EMBC), Osaka, JAPAN. Book Series: IEEE Engineering in Medicine \& Biology Society Conf Proceedings, 2013, pp: 3638-3641.

[5] Z.R. Parhi, S.K. Pradhan, A.K. Panda, and R.K. Behra, "The stable and precise motion control for multiple wheelchairs," Appl. Soft Comput vol 9(2), 2009, 477- 487.

[6] D. A. Sanders. B. Sanders, A. Gegov and D. Ndzi, "Results from investigating powered wheelchair users learning to drive with varying levels of sensor support". Proc SAI Intelligent Systems 17.

[7] K. T. Song, and C. C. Chen, "Application of asymmetric mapping for wheelchair navigation using ultrasonic sensors," J. Intell. Wheelchairic Syst., 1996, 17(3), 243-264.

[8] R. Huq, G. K. I. Mann and R. G. Gosine, "Wheelchair navigation using motor schema and fuzzy context dependent behaviour modulation," Appl. Soft Comput., 2008, 8(1), 422-436.

[9] M. Begum, G. K. I. Mann, and R. G. Gosine, "Integrated fuzzy logic and genetic algorithmic approach for simultaneous localization and mapping of wheelchairs," Appl. Soft Comput., 2008, 8(1), 150-165.

[10] M. Bennewitz and W. Burgard, "A probabilistic method for planning collision-free trajectories of multiple wheelchairs," In Proc 14th Euro Conf on AI, Berlin, Germany, 20-25 August 2000, pp. 9-15 (ECAI).

[11] W. Gueaieb and M. S. Miah, "An intelligent wheelchair navigation technique using RFID technology,” IEEE Trans. Instrum. Measmt, 2008, 57(9), 1908-1917. 
[12] C. L. Hwang and N. W. Chang, "Fuzzy decentralized sliding-mode control of a car-like wheelchair in distributed sensor-network spaces," IEEE Trans. Fuzzy Syst., 2008, 16(1), 97-109.

[13] A.V. Nguyen, and L.B. Nguyen, S. Su, H.T. Nguyen, "The Advancement of an Obstacle Avoidance Bayesian Neural Network for an Intelligent Wheelchair", 35th Int Conf of IEEE-Engineering-in-Medicine-andBiology-Society (EMBC), Osaka. Book Series: IEEE Eng in Medicine and Biology Society Conf Proc, 2013, pp: 3642-3645.

[14] D. A. Sanders. "Using a self-reliance factor for a disabled driver to decide on the share of combined-control between a powered wheelchair and an ultrasonic sensor system". IEEE Trans on Neural Systems \& Rehab' Engineering. DOI: 10.1109/TNSRE.2016.2620988

[15] D. Sanders, I. Stott, J. Graham-Jones, A. Gegov, and G.E. Tewkesbury, "Expert system to interpret hand tremor and provide joystick position signals for powered wheelchairs with ultrasonic sensor systems". Ind Rob 38 (6). 2011, pp. 585-598.

[16] D.C. Robinson, D.A. Sanders, and E. Mazharsolook. "Ambient intelligence for optimal manufacturing and energy efficiency". Assem. Autom. Vol 35 (3), 201, pp: 234-248

[17] D.A. Sanders, G. Tewkesbury, A. Gegov, et al. "Fast transformations to provide simple geometric models of moving objects". Proc' Intelligent Robotics \& Application Confs, Pt I Vol 9244, 2015, pp: 604-615.

[18] D. Sanders, M. Langner, and G. Tewkesbury, "Improving wheelchairdriving using a sensor system to control wheelchair-veer and variableswitches as an alternative to digital-switches or joysticks", Ind Rob: An int' jnl, 37 (2). 2010, pp. 151-167.

[19] J. Larsson M., Broxvall and A. Saffiotti, "Laser-based corridor detection for reactive Navigation. Ind Rob: An int' jnl, 35 (1), 2008, pp 69-79.

[20] D. Sanders, J. Graham-Jones, and A. Gegov, "Improving ability of tele operators to complete progressively more difficult mobile robot paths using simple expert systems and ultrasonic sensors," Ind Rob: An int' jnl, 37 (5). 2010, pp. 431-440.

[21] S. Lee "Use of infrared light reflecting landmarks for localization. Ind Rob: An int' jnl 36 (2), 2009, pp: 138-145.

[22] V. Milanes, J. Naranjo, C. Gonzalez, "Autonomous vehicle based in cooperative GPS and inertial systems", Robotica 26, pp 627-633. 2008

[23] D. Sanders and I. Stott, "A new prototype intelligent mobility system to assist powered wheelchair users", Ind Rob 26(6), 09, pp: 466-475.

[24] Y. C. Chang and Y. Yamamoto "On-line path planning strategy integrated with collision and dead-lock avoidance schemes for wheeled wheelchair in indoor environments," Ind Rob: An int' jnl, 35 (5), 2008, pp 421-434.

[25] D. Sanders, "Progress in machine intelligence", Ind Rob 35 (6), 2008, pp: 485-487.

[26] D. A. Sanders, G. Lambert, and L. Pevy, "Pre-locating corners in images in order to improve the extraction of Fourier descriptors and subsequent recognition of shipbuilding parts", Proc' Inst Mech Eng PART B-Jrnl of Engineering manufacture 223, vol 9, 2009, pp: 1217-1223.

[27] D. Sanders, "Comparing speed to complete progressively more difficult mobile robot paths between human tele-operators and humans with sensor-systems to assist," Assem. Autom. vol 29 (3), 2009, pp 230-248.

[28] D. Sanders, "Analysis of the effects of time delays on the teleoperation of a mobile robot in various modes of operation," Ind Rob vol 36 Issue: 6, 2009, pp: 570-584. 2.

[29] D. A. Sanders, M. Langner, A. E. Gegov, D. Ndzi, H. Sanders, and G. E. Tewkesbury, G, "Tele-operator performance and their perception of system time lags when completing mobile robot tasks", Proc 9th Int Conf on Human Systems Interaction, pp: 236-242. 2016.
[30] D. A. Sanders, D. L. Ndzi, S. Chester, and M. Malik, "Adjustment of teleoperator learning when provided with different levels of sensor support while driving mobile robots", IEEE Proc' of SAI Intelligent Systems, pp: 962-967. 2016

[31] D. A. Sanders and N.Bausch, (Ed H. Liu). "Improving Steering of a Powered Wheelchair Using an Expert System to Interpret Hand Tremor". Proc of Intelligent Wheelchairics and Applications (Icira 2015), Pt Ii Volume: 9245, pp: 460-471. 2015

[32] D. Sanders, I. J. Stott and D. C. Robinsosn et al. "Analysis of successes and failures with a tele-operated mobile robot in various modes of operation". Robotica 30, pp: 973-988. 2012.

[33] D. A. Sanders, G. E. Tewkesbury, and I. J. Stott, et al. "Simple expert systems to improve an ultrasonic sensor - system for a tele - operated mobile - robot”. Sensor Review 31 (3), pp 246-260. 2011.

[34] G. Eason, B. Noble, and I. N. Sneddon, “On certain integrals of LipschitzHankel type involving products of Bessel functions," Phil. Trans. Roy. Soc. London, vol. A247, pp. 529-551, April 1955

[35] D. A. Sanders, H. Sanders, D. L. Ndzi, and A. E. Gegov, "Rule-based system to assist a tele-operator with driving a mobile robot", IEEE Proc' of SAI Intelligent Systems Conf’, pp: 987 - 994. 2016.

[36] D. Sanders and A. Geov. "AI tools for use in Assembly Automation and some examples of recent applications. Assem. Autom. Vol 33 (2), pp: 184-194. 2013.

[37] D. A. Sanders, G. E. Tewkesbury, and D. Ndzi et al. "Improving automatic robotic welding in shipbuilding through the introduction of a corner-finding algorithm to help recognise shipbuilding parts". Journal of Marine Science and Technology Vol 17 (2), pp: 231-238. 2012.

[38] D. Sanders, G. Lambert, and J. Graham-Jones et al. "A robotic welding system using image processing techniques and a CAD model to provide information to a multi-intelligent decision module". Assem. Autom. Vol: 30 (4), pp: 323-332. 2010.

[39] D. A. Sanders, and G. E. Tewkesbury. "A pointer device for TFT display screens that determines position by detecting colours on the display using a colour sensor and an Artificial Neural Network". Displays Vol 30 (2), pp: 84-96. 2009.

[40] J. Bergasa-Suso, D. A. Sanders, and G. E. Tewkesbury. "Intelligent browser-based systems to assist Internet users". IEEE Transactions on Education Vol 48 (4), pp: 580-585. 2005.

[41] D. A. Sanders, Y. Tan, I Rogers et al. "An expert system for automatic design-for-assembly". Assem. Autom. 29 (4), pp: 378-388. 2009.

[42] D. A. Sanders, G. Lambert, and L. Pevy. "Pre-locating corners in images in order to improve the extraction of Fourier descriptors and subsequent recognition of shipbuilding parts". Proc of IMechE Part B-Journal of Engineering Manufacture 223 (9), pp: 1217-1223. 2009.

[43] D. Sanders. "Controlling the direction of walkie type forklifts and pallet jacks on sloping ground”. Assem. Autom. 28 (4), pp: 317-324. 2008.

[44] D. A. Sanders, J. Bergasa-Suso, R. Khusainov, A. Gegov, S. Chester, and N. Bausch. "Introducing dead bands within two-dimensional clusters of user data to improve data classification". Proc' of the IEEE 9th Int Conf on Human Systems Interaction, pp: 14-20. 2016.

[45] D. A Sanders"Progress in machine intelligence". Ind Rob: An int' jnl 35 (6), pp: 485-487. 2008.

[46] A. Geov, N. Gobalakrishnan and D. A. Sanders. "Rule base compression in fuzzy systems by filtration of non-monotonic rules". Journal of Intelligent \& Fuzzy Systems Vol 27 (4), pp: 2029-2043. 2014.

[47] D. A. Sanders. A. Gegov, B. Sanders, and D. Ndzi, "Using confidence factors to share control between a mobile robot tele-operater and ultrasonic sensors". Proc SAI Intelligent Systems 17. 